\title{
Limitations of functional neuroimaging for patient selection and surgical planning in glioma surgery
}

\author{
Tej D. Azad, MD, MS, ${ }^{1}$ and Hugues Duffau, MD, PhD² \\ 'Department of Neurosurgery, Johns Hopkins Hospital, Baltimore, Maryland; and ²Department of Neurosurgery, Hôpital Gui de
Chauliac, Montpellier, France
}

\begin{abstract}
The optimal surgical management of gliomas requires a balance between surgical cytoreduction and preservation of neurological function. Preoperative functional neuroimaging, such as functional MRI (fMRI) and diffusion tensor imaging (DTI), has emerged as a possible tool to inform patient selection and surgical planning. However, evidence that preoperative fMRI or DTI improves extent of resection, limits neurological morbidity, and broadens surgical indications in classically eloquent areas is lacking. In this review, the authors describe facets of functional neuroimaging techniques that may limit their impact on neurosurgical oncology and critically evaluate the evidence supporting fMRI and DTI for patient selection and operative planning in glioma surgery. The authors also propose alternative applications for functional neuroimaging in the care of glioma patients.
\end{abstract}

https://thejns.org/doi/abs/10.3171/2019.11.FOCUS19769

KEYWORDS diffusion tensor imaging; functional MRI; tractography; direct electrical stimulation

$\mathrm{E}$ XTENT of resection remains a critical determinant of oncological outcome for patients with high- and low-grade glioma. ${ }^{4,21,48}$ However, realization of the true benefit of neurosurgical resection requires a balance between surgical cytoreduction and preservation of neurological function. Multiple approaches have emerged in an effort to extend the neurosurgeon's ability to achieve maximal safe resection, including fluorescence guidance, ${ }^{50,59}$ direct electrical stimulation (DES), ${ }^{12,14,15,45}$ intraoperative imaging, $, 3,47,52$ and preoperative functional imaging, such as functional MRI (fMRI) and diffusion tensor imaging (DTI). ${ }^{1,39,58}$

Ongoing advances in technology have led to widespread interest in functional neuroimaging for the preoperative planning of glioma surgery. ${ }^{55}$ The motivation behind this interest can be traced directly to the hypothesis that more precise mapping of neurological function will improve extent of resection, mitigate morbidity, and broaden surgical indications for lesions in classically eloquent areas. However, data to support this hypothesis remain scarce.

While fMRI and DTI are excellent didactic and research tools, their clinical utility requires further evidence. The foundations of fMRI and DTI are well established and there are many reviews detailing the potential of func- tional neuroimaging in brain tumor surgery. $1,1,10,11,17,39,44$ Therefore, the objective of this review is to detail nuances of functional neuroimaging techniques that may limit the ability of these techniques to impact neurosurgical oncology and to critically appraise the evidence supporting fMRI and DTI in glioma surgery for patient selection and operative planning.

\section{Review of fMRI and DTI Clinical Utility fMRI for Patient Selection and Preoperative Planning}

The contrast mechanism of fMRI is the ratio of deoxyhemoglobin to oxyhemoglobin, known as the blood oxygen level-dependent (BOLD) signal. The BOLD signal serves as a proxy for neuronal activity and has been proposed for preoperative assessment of motor and language mapping. The patient is given a series of motor and language tasks to perform and changes in BOLD signal are measured to infer areas of functional activation. ${ }^{55}$ More recently, there has been interest in using resting-state fMRI (rs-fMRI) to perform preoperative mapping. Resting-state fMRI does not require patient participation and can be performed under general anesthesia to detect BOLD signal variation between multiple spatially and functionally distinct restingstate networks. ${ }^{28,63}$ 
However, integration of fMRI into preoperative planning carries limitations. A key concern is the sensitivity and specificity of the technique, most notably for language mapping. Giussani et al. ${ }^{19}$ examined this question by synthesizing data from studies that directly compared DES and fMRI for language localization in patients with brain tumors. The authors identified 9 studies, including 5 that reported sensitivity ranging from $59 \%$ to $100 \%$ and specificity from $0 \%$ to $97 \%$. A limitation of this synthetic study was the underlying heterogeneity of the studies, including surgical indication, magnet strength, and task protocol. Kuchcinski et al. ${ }^{26}$ remedied these limitations in a cohort of 40 glioma patients with 3-T fMRI. Preoperative fMRI was compared with the results of DES during awake glioma resection site-by-site using a cortical grid. Using DES as the reference, fMRI demonstrated a sensitivity and specificity of $37.1 \%$ and $83.4 \%$, respectively. While no statistically significant associations with false-negative fMRI signals were identified, oligodendroglioma subtype, tumor relative cerebral blood volume $(\mathrm{CBV})>1.5$, lower cortical CBV, and distance to the tumor were associated with false-positive discrepancies. Similar studies with smaller cohorts found higher sensitivity and specificity for motor mapping (85\%-88\% and $81 \%-87 \%)$ than language mapping (40\%-80\% and $74 \%-84 \%))^{2,23,37}$ A recent meta-analysis by Metwali et al. synthesized 8 studies ( 6 for language, 2 for motor) that directly compared fMRI and DES for brain tumor surgery. ${ }^{34}$ The mean sensitivity and specificity of fMRI for the detection of functional motor areas were $92 \%$ (range $87.5 \%-100 \%$ ) and $76 \%$ (range $68.1 \%-87.1 \%$ ), respectively. The mean sensitivity and specificity of fMRI for the detection of functional language areas were $80 \%$ (range 64\%-100\%) and 71.5\% (range 50\%-89\%), respectively. ${ }^{34}$ Another recent synthetic study by Weng et al. extended the results of prior meta-analyses by investigating the sensitivity and specificity on both a per-site basis (i.e., each DES stimulation site was considered a separate data point across all patients) and a per-patient basis. ${ }^{57}$ The persite pooled sensitivity and specificity were $67 \%(95 \% \mathrm{CI}$ $51 \%-80 \%$ ) and $55 \%$ (95\% CI 25\%-82\%), while the perpatient pooled sensitivity and specificity were $44 \%(95 \%$ CI $14 \%-78 \%$ ) and $80 \%$ (95\% CI 54\%-93\%)..$^{57}$

Advocates of rs-fMRI suggest that the resting-state networks elucidated by this technique represent intrinsic functional networks and thus can be relied upon to guide resection. However, evidence to support this hypothesis remains limited. Cochereau et al. ${ }^{9}$ investigated this premise in a cohort of 98 patients with diffuse low-grade glioma. The authors identified a significant association between resting-state BOLD signal fluctuations and functional cortical units as defined by DES. They also observed significant between-patient variability in mapping fidelity and an accuracy rate of approximately $80 \%$ in the detection of functionally relevant cortical sites. This finding is consistent with other studies that compared DES and rsfMRI. ${ }^{18,36,42,63}$

Taken together, fMRI and rs-fMRI currently appear inadequate for standalone preoperative cortical functional mapping, particularly as it pertains to language localization. A key reason that both fMRI and rs-fMRI may have limited sensitivity and specificity when compared to DES is that DES provides a more direct assessment of neuronal function while fMRI BOLD signal is inherently a proxy measure.

A key limitation of fMRI is that it does not offer the surgeon the ability to distinguish between compensable areas that can be resected and critical areas that should be surgically preserved. This can result in the underselection of patients for surgery ${ }^{49}$ and may increase the likelihood of partial or subtotal resection due to concern for violation of cortical areas deemed functional by fMRI. Southwell et al. reported a series of 58 glioma patients with unifocal supratentorial disease who underwent glioma resection guided by DES within 6 months of undergoing a brain biopsy of the same lesion at another institution. They achieved an average extent of resection of nearly $90 \%$ with no new postoperative neurological deficits. ${ }^{49}$ Their findings suggest that decision-making based solely on preoperative structural or functional imaging is likely inadequate, particularly for cortical lesions. Intraoperative DES, in contrast, offers the ability to accurately identify functional brain regions. ${ }^{12,14,15,45}$ The distinction between fMRI and DES is even more pronounced when considering subcortical functional mapping, where DES has demonstrated clinical utility ${ }^{16,20}$ Multiple studies have demonstrated a reduction in BOLD sensitivity $29,33,51$ and greater susceptibility to physiological noise ${ }^{22,56}$ when applying fMRI to subcortical mapping.

\section{DTI for Patient Selection and Preoperative Planning}

DTI enables visualization of white matter tracts, revealing infiltration and displacement by intracranial lesions in order to hypothetically inform surgical planning.,11,55 Recently, international, multicenter efforts have emerged to systematically validate DTI tractography. Maier-Hein et al..$^{30}$ described the results of an international tractography challenge involving 96 distinct submissions from 20 research groups using a data set with ground-truth white matter tracts. The findings of this effort lay bare the current limitations of using DTI for surgical planning of gliomas. While the authors found that many tractograms contained at least $90 \%$ of the ground-truth tracts, these tractograms included more invalid than valid bundles (i.e., a high falsepositive rate). Pujol et al. presented a similar study more directly related to glioma surgery ${ }^{41}$ Eight teams from international institutions reconstructed the corticospinal tract in cases of glioma adjacent to the motor cortex using multiple tractography approaches with results evaluated by neurosurgeons and DTI experts. A key conclusion from this important study was the marked inter-algorithm variability, both in the hemisphere containing the tumor and in the contralateral hemisphere. Given identical data sets, tractograms vary widely based on the reconstruction algorithm. ${ }^{41}$ An additional conclusion from this study was relatively poor performance in delineating lateral projections compared to medial projections, a finding replicated by Mandelli et al. ${ }^{31,41}$ A more recent international effort, the 3D Validation of Tractography with Experimental MRI challenge, provided three unique data sets, a physical phantom, and two ex vivo brain specimens to 9 research groups, garnering 176 distinct submissions. ${ }^{46}$ Schilling et al. concluded that the anatomical accuracy of tractography 
has not substantively improved despite significant advances in tractography algorithms and methods. ${ }^{46}$

A second set of studies compare preoperative DTI tract reconstructions with intraoperative subcortical stimulation. A direct validation of DTI was performed by Leclercq et al., in which the authors preoperatively reconstructed 4 white matter tracts in 10 glioma patients and evaluated these DTI tractograms against intraoperative subcortical language mapping. ${ }^{27}$ While positive stimulation sites correlated with DTI tractograms (in 17 of 21 sites), negative tractograms did not rule out the presence of a white matter tract. ${ }^{27}$ Ostrý et al. ${ }^{38}$ prospectively enrolled 25 patients with solitary supratentorial intracerebral lesions compressing or infiltrating the corticospinal tract. Pre- and intraoperative corticospinal tract tractography was compared with intraoperative subcortical language mapping by DES. They observed that intraoperative image distortion occurred in more than one-third of patients, rendering DTI unusable in those cases. The authors concluded that DES-based subcortical mapping remains superior to DTI. Prospective studies evaluating DTI-based mapping are rare. However, $\mathrm{Wu}$ et al. ${ }^{60}$ conducted a prospective study of 328 cerebral gliomas, randomizing patients to either DTI and 3D MRI $(\mathrm{n}=118)$ or routine neuronavigation $(\mathrm{n}=120)$. In the DTI study arm they observed a significantly higher rate of gross-total resection among high-grade glioma patients and higher Karnofsky Performance Scale scores for high- and low-grade glioma patients. However, this study has important limitations. Specifically, the increased gross-total resection rate was observed only for high-grade tumors, the routine neuronavigation in the control arm did not utilize DES, and the study chiefly reported motor function outcomes. Future prospective studies should build on the work of Wu et al. by reporting more comprehensive functional outcomes and using DES-guided resection as the control arm.

Taken together, these studies indicate that DTI is fundamentally a structural imaging modality and less a tool for functional interrogation. Surgical planning driven solely by tractography would be at risk of offering unnecessarily limited resection, as the surgeon may decide to curtail his or her operative plan in an effort to spare white matter tracts. Additionally, it may drive underselection of patients for surgery, as preoperative tractography may falsely convince the surgeon, or the patient, that resection would lead to undue morbidity.

\section{Discussion}

Given the methodological limitations discussed above, we maintain that functional neuroimaging in its current form is inadequate for patient selection and surgical planning in glioma surgery. However, there are opportunities to improve and expand the utility of functional neuroimaging. First, it must be noted that the clinical studies discussed above may not be using technologies and algorithms at the bleeding edge of functional neuroimaging. As advances in functional neuroimaging continue to manifest, it remains possible that fMRI and DTI may be better poised to inform patient selection and surgical planning. Second, the integration of multiple noninvasive map- ping techniques may extend the promise of any individual technique. Future studies combining multiple techniques are likely nascent.

We posit that there may be intrinsic value to these techniques, if applied to the correct use case. Commonly reported outcome measures following glioma surgery account for motor and language function. However, quality of life after glioma resection must be considered broad$\mathrm{ly}^{24,53}$ and take into account higher-order neurological functions such as mentalizing/theory of mind, ${ }^{5,6}$ attentional processing, ${ }^{25,35}$ and executive function. It is in the elucidation of these higher-order functions that functional neuroimaging is most likely to advance the neurosurgical management of glioma patients. Recent work suggests that rs-fMRI ${ }^{61}$ and DTI, ${ }^{62}$ in conjunction with DES, may be able to map the networks and tracts involved in mentalizing tasks. Mandonnet et al. similarly used rs-fMRI and DTI pre- and postoperatively to illustrate that resection of a right temporoparietal glioma disrupted a large-scale network involved in cognitive flexibility. ${ }^{32}$ This paradigm of pre- and postoperative functional neuroimaging could be extended to develop strategies of monitoring neuroplasticity in glioma surgery, as demonstrated by several longitudinal functional neuroimaging studies. $3,8,13,54$

We posit that longitudinal functional neuroimaging of glioma patients would have both didactic and clinical utility. Not only would we extend our understanding of network-level neuroplasticity, but we could also use this information to personalize future neurosurgical treatment of individual patients. This could allow for the evolution of iterative, multistage surgical strategies ${ }^{40}$ that move fundamentally closer to the goal of extending neurooncological survival through more complete resection while preserving neurological function.

\section{Conclusions}

Technological progress has fundamentally advanced neuroimaging, enabling functional neuroimaging to continue to extend our understanding of the CNS. However, these tools are derived from cohort-level statistical models and are difficult to apply to individual surgical candidates. We agree that advances in algorithms produce functional neuroimaging techniques that may be useful adjuncts to intraoperative DES but remain skeptical that these modalities meaningfully impact glioma surgery. To date, evidence that preoperative DTI or fMRI improves extent of resection, minimizes morbidity, and broadens surgical indications in classically eloquent areas remains scarce. Further work should focus on carefully designed prospective comparative studies and on longitudinal functional neuroimaging studies to better understand, and possibly clinically apply, neuroplasticity in glioma patients.

\section{References}

1. Abhinav K, Yeh FC, Mansouri A, Zadeh G, FernandezMiranda JC: High-definition fiber tractography for the evaluation of perilesional white matter tracts in high-grade glioma surgery. Neuro Oncol 17:1199-1209, 2015

2. Bizzi A, Blasi V, Falini A, Ferroli P, Cadioli M, Danesi U, et al: Presurgical functional MR imaging of language and mo- 
tor functions: validation with intraoperative electrocortical mapping. Radiology 248:579-589, 2008

3. Boyer A, Deverdun J, Duffau H, Le Bars E, Molino F, Menjot de Champfleur N, et al: Longitudinal changes in cerebellar and thalamic spontaneous neuronal activity after wideawake surgery of brain tumors: a resting-state fMRI study. Cerebellum 15:451-465, 2016

4. Brown TJ, Brennan MC, Li M, Church EW, Brandmeir NJ, Rakszawski KL, et al: Association of the extent of resection with survival in glioblastoma: a systematic review and metaanalysis. JAMA Oncol 2:1460-1469, 2016

5. Brüne M, Brüne-Cohrs U: Theory of mind-evolution, ontogeny, brain mechanisms and psychopathology. Neurosci Biobehav Rev 30:437-455, 2006

6. Campanella F, Fabbro F, Ius T, Shallice T, Skrap M: Acute effects of surgery on emotion and personality of brain tumor patients: surgery impact, histological aspects, and recovery. Neuro Oncol 17:1121-1131, 2015

7. Castellano A, Cirillo S, Bello L, Riva M, Falini A: Functional MRI for surgery of gliomas. Curr Treat Options Neurol 19:34, 2017

8. Charras P, Herbet G, Deverdun J, de Champfleur NM, Duffau $\mathrm{H}$, Bartolomeo P, et al: Functional reorganization of the attentional networks in low-grade glioma patients: a longitudinal study. Cortex 63:27-41, 2015

9. Cochereau J, Deverdun J, Herbet G, Charroud C, Boyer A, Moritz-Gasser S, et al: Comparison between resting state fMRI networks and responsive cortical stimulations in glioma patients. Hum Brain Mapp 37:3721-3732, 2016

10. Conti Nibali M, Rossi M, Sciortino T, Riva M, Gay LG, Pessina F, et al: Preoperative surgical planning of glioma: limitations and reliability of fMRI and DTI tractography. J Neurosurg Sci 63:127-134, 2019

11. Costabile JD, Alaswad E, D'Souza S, Thompson JA, Ormond DR: Current applications of diffusion tensor imaging and tractography in intracranial tumor resection. Front Oncol 9:426, 2019

12. De Witt Hamer PC, Robles SG, Zwinderman AH, Duffau $\mathrm{H}$, Berger MS: Impact of intraoperative stimulation brain mapping on glioma surgery outcome: a meta-analysis. J Clin Oncol 30:2559-2565, 2012

13. Deverdun J, van Dokkum LEH, Le Bars E, Herbet G, Mura T, D'agata B, et al: Language reorganization after resection of low-grade gliomas: an fMRI task based connectivity study. Brain Imaging Behav [epub ahead of print], 2019

14. Duffau H: Stimulation mapping of white matter tracts to study brain functional connectivity. Nat Rev Neurol 11:255265, 2015

15. Duffau H, Lopes M, Arthuis F, Bitar A, Sichez JP, Van Effenterre R, et al: Contribution of intraoperative electrical stimulations in surgery of low grade gliomas: a comparative study between two series without (1985-96) and with (19962003) functional mapping in the same institution. J Neurol Neurosurg Psychiatry 76:845-851, 2005

16. Duffau H, Peggy Gatignol ST, Mandonnet E, Capelle L, Taillandier L: Intraoperative subcortical stimulation mapping of language pathways in a consecutive series of 115 patients with Grade II glioma in the left dominant hemisphere. J Neurosurg 109:461-471, 2008

17. Essayed WI, Zhang F, Unadkat P, Cosgrove GR, Golby AJ, O'Donnell LJ: White matter tractography for neurosurgical planning: a topography-based review of the current state of the art. Neuroimage Clin 15:659-672, 2017

18. Fox MD, Qian T, Madsen JR, Wang D, Li M, Ge M, et al: Combining task-evoked and spontaneous activity to improve pre-operative brain mapping with fMRI. Neuroimage 124 (Pt A):714-723, 2016

19. Giussani C, Roux FE, Ojemann J, Sganzerla EP, Pirillo D, Papagno C: Is preoperative functional magnetic resonance imaging reliable for language areas mapping in brain tumor surgery? Review of language functional magnetic resonance imaging and direct cortical stimulation correlation studies. Neurosurgery 66:113-120, 2010

20. Han SJ, Morshed RA, Troncon I, Jordan KM, Henry RG, Hervey-Jumper SL, et al: Subcortical stimulation mapping of descending motor pathways for perirolandic gliomas: assessment of morbidity and functional outcome in 702 cases. J Neurosurg 131:201-208, 2018

21. Hervey-Jumper SL, Berger MS: Role of surgical resection in low- and high-grade gliomas. Curr Treat Options Neurol 16:284, 2014

22. Hutton C, Josephs O, Stadler J, Featherstone E, Reid A, Speck O, et al: The impact of physiological noise correction on fMRI at 7 T. Neuroimage 57:101-112, 2011

23. Ille S, Sollmann N, Hauck T, Maurer S, Tanigawa N, Obermueller $\mathrm{T}$, et al: Combined noninvasive language mapping by navigated transcranial magnetic stimulation and functional MRI and its comparison with direct cortical stimulation. J Neurosurg 123:212-225, 2015

24. Klein M: Neurocognitive functioning in adult WHO grade II gliomas: impact of old and new treatment modalities. Neuro Oncol 14 (Suppl 4):iv17-iv24, 2012

25. Kollndorfer K, Furtner J, Krajnik J, Prayer D, Schöpf V: Attention shifts the language network reflecting paradigm presentation. Front Hum Neurosci 7:809, 2013

26. Kuchcinski G, Mellerio C, Pallud J, Dezamis E, Turc G, Rigaux-Viodé $\mathrm{O}$, et al: Three-tesla functional MR language mapping: comparison with direct cortical stimulation in gliomas. Neurology 84:560-568, 2015

27. Leclercq D, Duffau H, Delmaire C, Capelle L, Gatignol P, Ducros M, et al: Comparison of diffusion tensor imaging tractography of language tracts and intraoperative subcortical stimulations. J Neurosurg 112:503-511, 2010

28. Leuthardt EC, Guzman G, Bandt SK, Hacker C, Vellimana AK, Limbrick D, et al: Integration of resting state functional MRI into clinical practice-a large single institution experience. PLoS One 13:e0198349, 2018

29. Lutti A, Thomas DL, Hutton C, Weiskopf N: High-resolution functional MRI at $3 \mathrm{~T}$ : 3D/2D echo-planar imaging with optimized physiological noise correction. Magn Reson Med 69:1657-1664, 2013

30. Maier-Hein KH, Neher PF, Houde JC, Côté MA, Garyfallidis E, Zhong J, et al: The challenge of mapping the human connectome based on diffusion tractography. Nat Commun 8:1349, 2017

31. Mandelli ML, Berger MS, Bucci M, Berman JI, Amirbekian B, Henry RG: Quantifying accuracy and precision of diffusion MR tractography of the corticospinal tract in brain tumors. J Neurosurg 121:349-358, 2014

32. Mandonnet E, Cerliani L, Siuda-Krzywicka K, Poisson I, Zhi N, Volle E, et al: A network-level approach of cognitive flexibility impairment after surgery of a right temporo-parietal glioma. Neurochirurgie 63:308-313, 2017

33. Marquis R, Muller S, Lorio S, Rodriguez-Herreros B, MelieGarcia L, Kherif F, et al: Spatial resolution and imaging encoding fMRI settings for optimal cortical and subcortical motor somatotopy in the human brain. Front Neurosci 13:571, 2019

34. Metwali H, Raemaekers M, Kniese K, Kardavani B, Fahlbusch R, Samii A: Reliability of functional magnetic resonance imaging in patients with brain tumors: a critical review and meta-analysis. World Neurosurg 125:183-190, 2019

35. Mickleborough MJ, Kelly ME, Gould L, Ekstrand C, Lorentz E, Ellchuk T, et al: Inclusion of attentional networks in the pre-surgical neuroimaging assessment of a large deep hemispheric cavernous malformation: an FMRI case report. Cerebrovasc Dis 39:202-208, 2015 
36. Mitchell TJ, Hacker CD, Breshears JD, Szrama NP, Sharma M, Bundy DT, et al: A novel data-driven approach to preoperative mapping of functional cortex using resting-state functional magnetic resonance imaging. Neurosurgery 73:969-983, 2013

37. Morrison MA, Tam F, Garavaglia MM, Hare GM, Cusimano MD, Schweizer TA, et al: Sources of variation influencing concordance between functional MRI and direct cortical stimulation in brain tumor surgery. Front Neurosci 10:461, 2016

38. Ostrý S, Belšan T, Otáhal J, Beneš V, Netuka D: Is intraoperative diffusion tensor imaging at 3.0T comparable to subcortical corticospinal tract mapping? Neurosurgery 73:797-807, 2013

39. Ottenhausen M, Krieg SM, Meyer B, Ringel F: Functional preoperative and intraoperative mapping and monitoring: increasing safety and efficacy in glioma surgery. Neurosurg Focus 38(1):E3, 2015

40. Picart T, Herbet G, Moritz-Gasser S, Duffau H: Iterative surgical resections of diffuse glioma with awake mapping: how to deal with cortical plasticity and connectomal constraints? Neurosurgery 85:105-116, 2019

41. Pujol S, Wells W, Pierpaoli C, Brun C, Gee J, Cheng G, et al: The DTI Challenge: Toward standardized evaluation of diffusion tensor imaging tractography for neurosurgery. J Neuroimaging 25:875-882, 2015

42. Qiu TM, Yan CG, Tang WJ, Wu JS, Zhuang DX, Yao CJ, et al: Localizing hand motor area using resting-state fMRI: validated with direct cortical stimulation. Acta Neurochir (Wien) 156:2295-2302, 2014

43. Rao G: Intraoperative MRI and maximizing extent of resection. Neurosurg Clin N Am 28:477-485, 2017

44. Salama GR, Heier LA, Patel P, Ramakrishna R, Magge R, Tsiouris AJ: Diffusion weighted/tensor imaging, functional MRI and perfusion weighted imaging in glioblastoma-foundations and future. Front Neurol 8:660, 2018

45. Sanai N, Mirzadeh Z, Berger MS: Functional outcome after language mapping for glioma resection. N Engl J Med 358:18-27, 2008

46. Schilling KG, Nath V, Hansen C, Parvathaneni P, Blaber J, Gao Y, et al: Limits to anatomical accuracy of diffusion tractography using modern approaches. Neuroimage 185:1-11, 2019

47. Senft C, Seifert V, Hermann E, Franz K, Gasser T: Usefulness of intraoperative ultra low-field magnetic resonance imaging in glioma surgery. Neurosurgery 63 (4 Suppl 2):257-267, 2008

48. Smith JS, Chang EF, Lamborn KR, Chang SM, Prados MD, $\mathrm{Cha} S$, et al: Role of extent of resection in the long-term outcome of low-grade hemispheric gliomas. J Clin Oncol 26:1338-1345, 2008

49. Southwell DG, Birk HS, Han SJ, Li J, Sall JW, Berger MS: Resection of gliomas deemed inoperable by neurosurgeons based on preoperative imaging studies. J Neurosurg 129:567-575, 2018

50. Stummer W, Stocker S, Wagner S, Stepp H, Fritsch C, Goetz $\mathrm{C}$, et al: Intraoperative detection of malignant gliomas by 5-aminolevulinic acid-induced porphyrin fluorescence. Neurosurgery 42:518-526, 1998

51. Triantafyllou C, Hoge RD, Krueger G, Wiggins CJ, Potthast A, Wiggins GC, et al: Comparison of physiological noise at $1.5 \mathrm{~T}, 3 \mathrm{~T}$ and $7 \mathrm{~T}$ and optimization of fMRI acquisition parameters. Neuroimage 26:243-250, 2005

52. Unsgaard G, Selbekk T, Brostrup Müller T, Ommedal S, Torp SH, Myhr G, et al: Ability of navigated 3D ultrasound to delineate gliomas and metastases-comparison of image interpretations with histopathology. Acta Neurochir (Wien) 147:1259-1269, 2005
53. van Kessel E, Baumfalk AE, van Zandvoort MJE, Robe PA, Snijders TJ: Tumor-related neurocognitive dysfunction in patients with diffuse glioma: a systematic review of neurocognitive functioning prior to anti-tumor treatment. J Neurooncol 134:9-18, 2017

54. Vassal M, Charroud C, Deverdun J, Le Bars E, Molino F, Bonnetblanc F, et al: Recovery of functional connectivity of the sensorimotor network after surgery for diffuse low-grade gliomas involving the supplementary motor area. J Neurosurg 126:1181-1190, 2017

55. Villanueva-Meyer JE, Mabray MC, Cha S: Current clinical brain tumor imaging. Neurosurgery 81:397-415, 2017

56. Viviani R: A digital atlas of middle to large brain vessels and their relation to cortical and subcortical structures. Front Neuroanat 10:12, 2016

57. Weng HH, Noll KR, Johnson JM, Prabhu SS, Tsai YH, Chang SW, et al: Accuracy of presurgical functional MR imaging for language mapping of brain tumors: a systematic review and meta-analysis. Radiology 286:512-523, 2018

58. Wengenroth M, Blatow M, Guenther J, Akbar M, Tronnier VM, Stippich C: Diagnostic benefits of presurgical fMRI in patients with brain tumours in the primary sensorimotor cortex. Eur Radiol 21:1517-1525, 2011

59. Widhalm G, Olson J, Weller J, Bravo J, Han SJ, Phillips J, et al: The value of visible 5-ALA fluorescence and quantitative protoporphyrin IX analysis for improved surgery of suspected low-grade gliomas. J Neurosurg [epub ahead of print May 10, 2019. DOI: 10.3171/2019.1.JNS182614]

60. Wu JS, Zhou LF, Tang WJ, Mao Y, Hu J, Song YY, et al: Clinical evaluation and follow-up outcome of diffusion tensor imaging-based functional neuronavigation: a prospective, controlled study in patients with gliomas involving pyramidal tracts. Neurosurgery 61:935-949, 2007

61. Yordanova YN, Cochereau J, Duffau H, Herbet G: Combining resting state functional MRI with intraoperative cortical stimulation to map the mentalizing network. Neuroimage 186:628-636, 2019

62. Yordanova YN, Duffau H, Herbet G: Neural pathways subserving face-based mentalizing. Brain Struct Funct 222:3087-3105, 2017

63. Zhang D, Johnston JM, Fox MD, Leuthardt EC, Grubb RL, Chicoine MR, et al: Preoperative sensorimotor mapping in brain tumor patients using spontaneous fluctuations in neuronal activity imaged with functional magnetic resonance imaging: initial experience. Neurosurgery 65 (6 Suppl):226-236, 2009

\section{Disclosures}

The authors report no conflict of interest concerning the materials or methods used in this study or the findings specified in this paper.

\section{Author Contributions}

Conception and design: both authors. Acquisition of data: both authors. Analysis and interpretation of data: both authors. Drafting the article: both authors. Critically revising the article: both authors. Reviewed submitted version of manuscript: both authors. Administrative/technical/material support: Duffau. Study supervision: Duffau.

\section{Correspondence}

Tej D. Azad: Johns Hopkins Hospital, Baltimore, MD. tazad1@ jhmi.edu. 